\title{
On alternative methods of determining Radius of Curvature using Newton's Rings set up
}

\author{
K. H. Raveesha ${ }^{1 \star}$, Kamal Kumar ${ }^{2}$, B. K. Prasad ${ }^{3}$ \\ ${ }^{1}$ Dept. of Physics, CMR Institute of Technology, Bangalore \\ ${ }^{2}$ Dept. of Mathematics, CMR Institute of Technology, Bangalore \\ ${ }^{3}$ Dept of Physics, CMR Institute of Technology, Bangalore \\ *E-mail address: ${ }^{1}$ hod.physics@cmrit.ac.in, ${ }^{2}$ Kamal.k@cmrit.ac.in, \\ 3Prasad.bk@cmrit.ac.in
}

\begin{abstract}
It well known that radius of curvature of a plano convex lens can be determined using Newton's Rings set up making use of Interference by division of amplitude principle. The general method widely used involves measurement of diameter of several circular dark fringes. We propose two alternative methods involving different formulae. It is based on the fact that, the formation of bright and dark interference fringes is a measure of the thickness of the air film at that point. The methods use simpler geometry and the formulae are easier to derive. We present the experimental data. The results from the experiment are in agreement with results obtained through the general method.
\end{abstract}

Keywords: Plano convex lens; Interference; fringes; destructive interference; constructive interference

\section{INTRODUCTION}

It is well known Newton described the formation of bright and dark rings using the plano convex lens [1]. It is understood that when a monochromatic beam of light falls normally on a Plano-convex lens kept on an optically plane glass plate, concentric bright and dark circular fringes are formed due to the constructive and destructive interference of light waves reflected from the lower surface of the lens and the upper surface of the plane glass plate. Formation of a bright or dark fringe at a point depends on the thickness of the air film at that point (which creates path difference)[2]. Conventionally Newton's rings experiment is used to determine the radius of curvature of a plano convex lens. The general method widely used involves measurement of the diameter of several fringes. It is described in the section 2 . We have described two alternative methods to determine radius of curvature in section $3 \& 4$ with detailed experimental data and analysis. The results obtained by the alternative methods are in close agreement with that obtained through the general method. Conclusions are presented in section 5 . 


\section{GENERAL METHOD TO FIND RADIUS OF CURVATURE OF PLANO CONVEX LENS}

Setting up the Newtons rings, the diameter of $2^{\text {nd }}, 4^{\text {th }}, 6^{\text {th }}, 8^{\text {th }}, 10^{\text {th }}$ and $12^{\text {th }}$ dark fringes are calculated. If $d_{m}$ and $d_{n}$ represent the diameter of the $m^{\text {th }}$ and $n^{\text {th }}$ dark fringe, the radius of curvature is determined using the formula

$$
R=\frac{d_{m}^{2}-d_{n}^{2}}{4 .(m-n) \lambda}
$$

For the given planoconvex lens, the value of $\mathrm{R}$ is found through this method is $0.93 \mathrm{~m}$ (manufacturer has prescribed the value as $1 \mathrm{~m}$ ).

\section{ALTERNATIVE METHOD 1 TO FIND RADIUS OF CURVATURE}

This method involves the measurement of radius of a clearly visible dark fringe preferable away from the centre. By knowing the fringe number, the thickness of the air film which creates the path difference causing the destructive interference leading to the formation of that dark fringe is calculated. Radius of curvature is obtained from the formula which is easily derived from trignametrical relationships. It is assumed that the radius of curvature is large in comparison with the path difference.

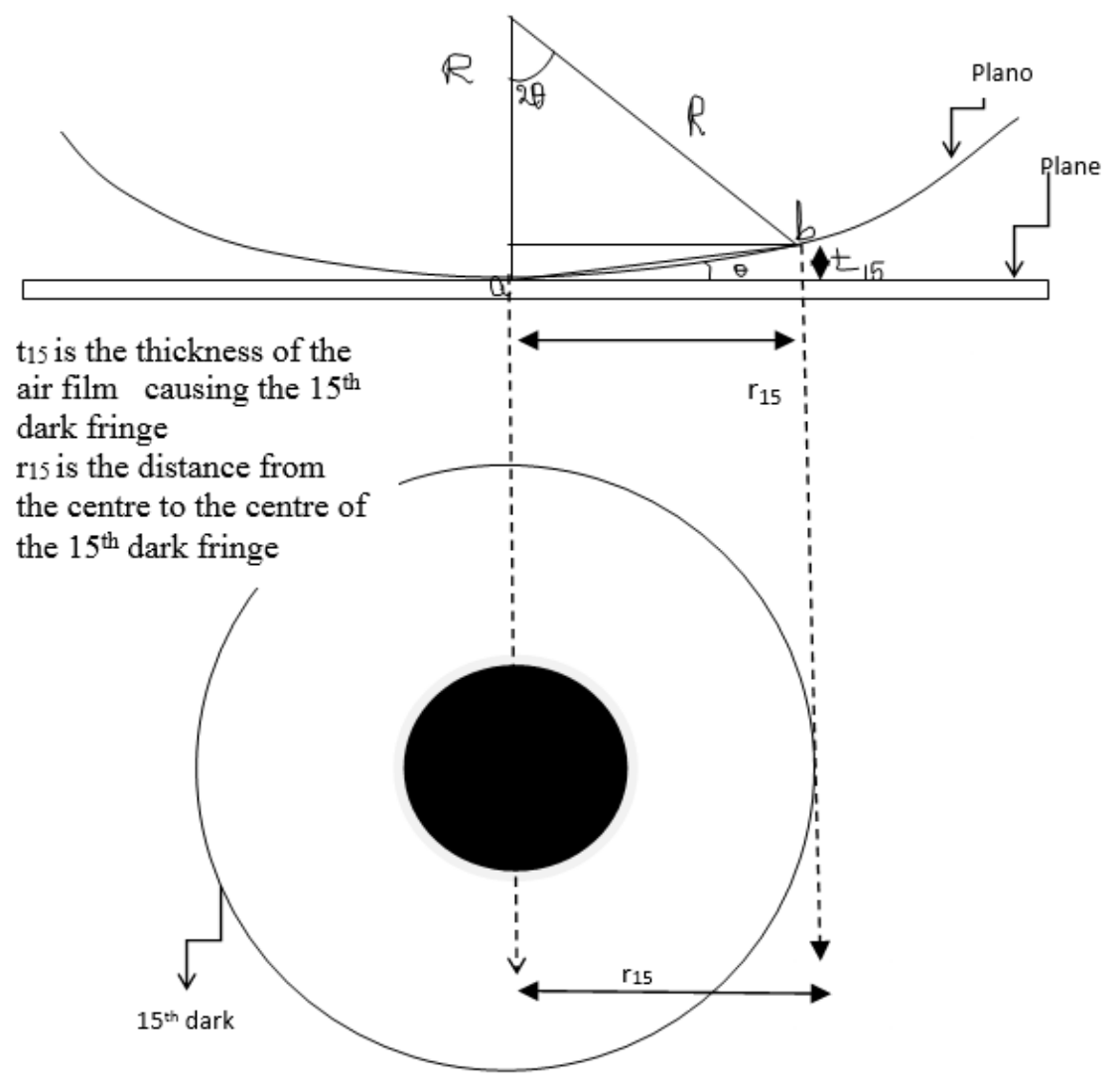

Figure 1. Newton's rings set up. 


\section{Theory}

From the figure (1),

$$
\begin{aligned}
& r_{15}^{2}+t_{15}^{2}=(\overline{a b})^{2} \\
& \overline{a b}=\sqrt{r_{15}^{2}+t_{15}^{2}} \\
& \theta=\tan ^{-1}\left(\frac{t_{15}}{r_{15}}\right)
\end{aligned}
$$

From trignametry, ssuming $\overline{a b}$ to be very small,

$$
\begin{aligned}
& R .2 \theta=\overline{a b} \\
& R=\frac{\overline{a b}}{2 \theta}
\end{aligned}
$$

where $\mathrm{R}$ is the radius of curvature of the plano convex lens

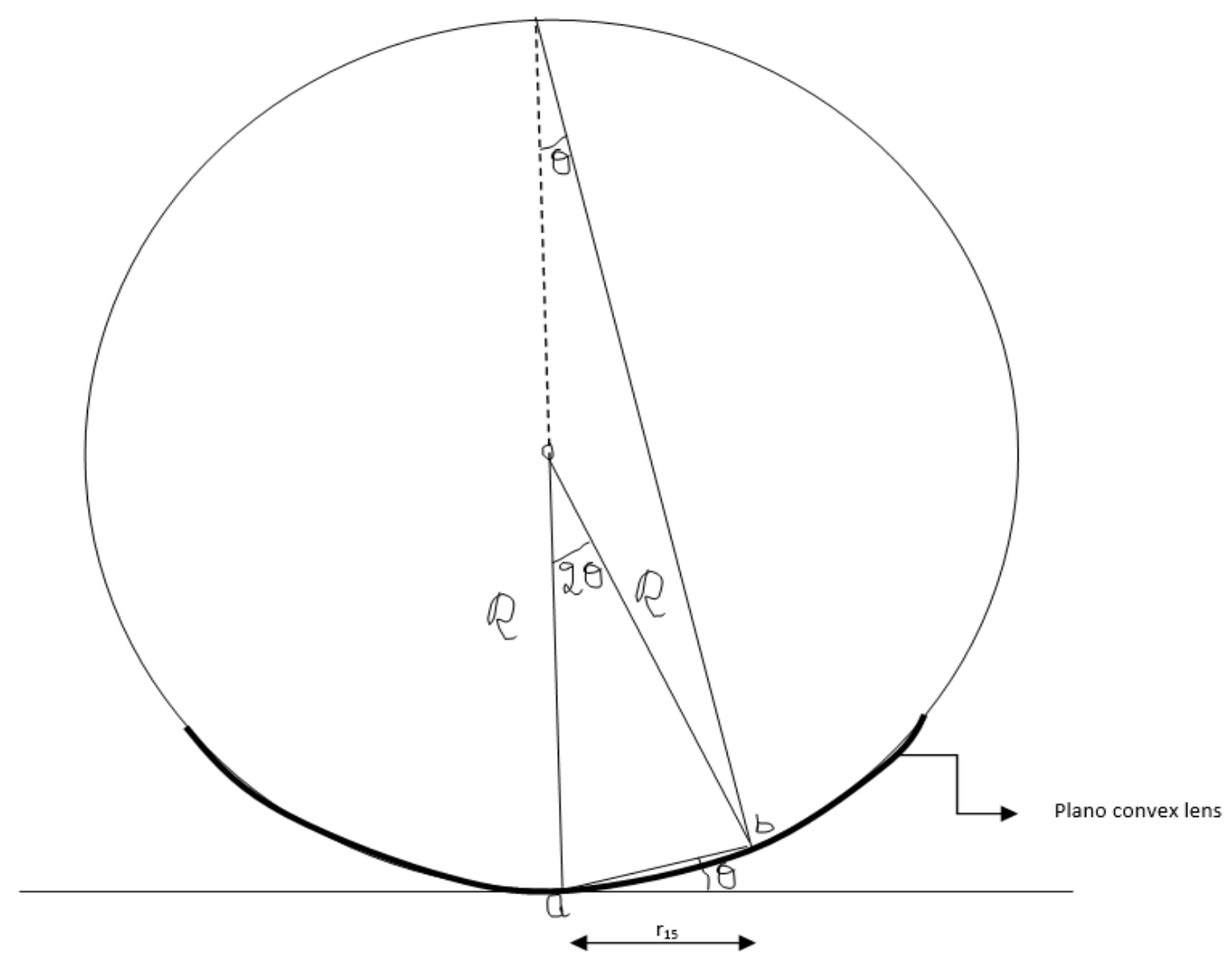

Figure 2. Circle geometry. 


\section{Experimental data, analysis and results}

From the condition for the formation of dark fringe (destructive interference)

$$
\begin{aligned}
2 t+\frac{\lambda}{2} & =(2 n+1) \frac{\lambda}{2} \\
t & =\frac{n \lambda}{2}
\end{aligned}
$$

where $t$ is the thickness of the air film causing the formation of dark fringe $\lambda$ is the wavelength of light used in the experiment $\left(5896 \times 10^{-10} \mathrm{~m}\right)$

For $15^{\text {th }}$ dark fringe, $\mathrm{n}=15$

$$
t_{15}=15 \times \frac{5896 \times 10^{-10}}{2}=4.422 \times 10^{-6} \mathrm{~m}
$$

Distance from the centre to the $15^{\text {th }}$ dark fringe in the experiment is

$$
\mathrm{r}_{15}=2.90 \times 10^{-3} \mathrm{~m}
$$

From (2),

$$
\overline{a b}=\sqrt{\left(2.9 \times 10^{-3}\right)^{2}+\left(4.422 \times 10^{-6}\right)^{2}}=0.0029 m
$$

From $(3), \quad \theta=\tan ^{-1}\left(\frac{4.422 \times 10^{-6}}{2.9 \times 10^{-3}}\right)=0.087^{0}=0.00151$ radian

From (4), Radius of curvature $\quad R=\frac{0.0029}{2 \times 0.00151}=0.96 \mathrm{~m}$

Where as we have considered the $15^{\text {th }}$ dark fringe for our calculations, any clearly visible fringe could be considered.

\section{ALTERNATIVE METHOD 2 TO FIND RADIUS OF CURVATURE}

This involves identifying a clearly visible dark fringe (say $15^{\text {th }}$ dark fringe as in the previous case) and calculating the thickness of air film creating the path difference causing the formation of dark fringe. The formula used is derived from using geometrical relationships.

From figure (1)

$$
\left(R-t_{15}\right)^{2}+r_{15}^{2}=R^{2}
$$

Simplification yields 
Radius of curvature

$$
R=\frac{t_{15}^{2}+r_{15}^{2}}{2 t_{15}^{2}}
$$

For $15^{\text {th }}$ dark fringe, $\mathrm{n}=15$,

From (5) \& (6)

$$
t_{15}=4.422 \times 10^{-6} \mathrm{~m}
$$

Distance from the centre to the $15^{\text {th }}$ dark fringe in the experiment is $\mathrm{r}_{15}=2.90 \times 10^{-3} \mathrm{~m}$

Substituting these parameters in (7)

$$
\begin{aligned}
& R=\frac{\left(4.422 \times 10^{-6}\right)^{2}+\left(2.90 \times 10^{-3}\right)^{2}}{8.844 \times 10^{-6}} \\
& R=0.95 m
\end{aligned}
$$

It may be noted that the radius of curvature obtained from the alternative methods (1) \& (2) are in close agreement $(0.96 \mathrm{~m} \& 0.95 \mathrm{~m}$ respectively) with the result obtained through the general method $(0.93 \mathrm{~m})$.

\section{CONCLUSIONS}

Two alternative methods to determine the radius of curvature are presented. The results obtained are in close agreement. The two proposed methods are relatively easier as they involve measurement related to one fringe as compared to measurements related to 6 fringes in the general method. Based on the authors interactions with several student groups at undergraduate level, it may be mentioned that students while conducting these experimental methods are able to develop an appreciation on how trigonometry can be utilized to develop simpler alternative methods. These alternative methods may be particularly useful in Industrial situations where radius of curvature many plano convex lenses are to determined in a short duration of time as these methods involve lesser measurements (one fringe) and hence automation is easier.

\section{Acknowledgements}

The authors are deeply indebted to the authorities of CMR Institute of Technology, Bangalore for offering necessary laboratory and library facilities which resulted in this research work.

\section{References}

[1] Ajoy Ghatak, 1991, Optics ,Tata Mcgraw Hill publication, $3^{\text {rd }}$ Edition, 13.16

[2] Chattopadhyay, 2002, An advanced course in Practical Physics, Central Publication, $6^{\text {th }}$ Edition, 227 